Meta

Journal des traducteurs

Translators' Journal

\title{
Quand les paroles ne s'envolent plus et que les murs ont des oreilles
}

\section{Robert Dubuc}

Volume 16, numéro 4, décembre 1971

URI : https://id.erudit.org/iderudit/002640ar

DOI : https://doi.org/10.7202/002640ar

Aller au sommaire du numéro

Éditeur(s)

Les Presses de l'Université de Montréal

ISSN

0026-0452 (imprimé)

1492-1421 (numérique)

Découvrir la revue

Citer cet article

Dubuc, R. (1971). Quand les paroles ne s'envolent plus et que les murs ont des oreilles. Meta, 16(4), 222-224. https://doi.org/10.7202/002640ar d'utilisation que vous pouvez consulter en ligne.

https://apropos.erudit.org/fr/usagers/politique-dutilisation/ 


\section{PROBLÈMES ET SOLUTIONS}

\section{QUAND LES PAROLES NE S'ENVOLEN'T PLUS ET QUE LES MURS ONT DES OREILLES}

Le vocabulaire des microphones ne fait en général pas partie de l'arsenal lexicologique du traducteur généraliste, mais la généralisation des techniques audio-visuelles, les surprises de l'espionnage électronique et la multiplication des procédés de conservation du son lui donnent pourtant de plus en plus d'actualité.

Ce vocabulaire, qui tient à la fois de l'acoustique et de l'électronique, ne se distingue guère des autres vocabulaires techniques existants : il a ses expressions familières : mike, micro, hum, ronflement; ses tours savants : cardioïde, hypercardioïde; ses synonymes, qu'il n'est pas toujours facile de démêler.

C'est à ce dernier problème qu'il convient de s'arrêter en prenant comme point de repère les trois types fondamentaux de microphones les plus répandus dans la radiotélévision nord-américaine ${ }^{1}$.

Il y a d'abord les ribbon microphones, caractérisés par le déplacement d'un ruban métallique ondulé dans le champ d'un aimant permanent. Cette expression se traduit «providentiellement » en français par microphone à ruban ${ }^{2}$. Si d'aventure vous tombez sur l'expression désuète band microphone, sachez y reconnaître un pur synonyme de ribbon microphone pour éviter le piège d'une traduction littérale.

L'expression dynamic microphone recèle elle aussi un piège pour celui qui traduit littéralement. On aurait souhaité établir entre dynamic microphone et «microphone dynamique » la même égalité qu'entre ribbon et «à ruban ». Mais rien n'est jamais aussi simple. Ce qu'on nomme en Amérique du Nord dynamic microphone ${ }^{3}$ correspond en Grande-Bretagne à moving-coil microphone et doit se rendre en français par microphone à bobine mobile. Ce microphone est le

1. Engineering Headquarters, TB125, Broadcast Microphones, Montreal, Canadian Broadcasting Corporation, $1968,103 \mathrm{p}$.

2. H. Piraux, Dictionnaire général d'acoustique et d'électroacoustique, Paris, Eyrolles, 1964, p. 189.

3. Engineering Headquarters, op. cit., p. 3 et 11. 
cousin germain du microphone à ruban et fonctionne selon le même principe, sauf que le ruban y est remplacé par une bobine. Cette famille de microphones, caractérisée par le déplacement d'un conducteur dans un champ magnétique, porte couramment le nom d'électrodynamique, mais devrait s'appeler, selon un édit de la Commission électrotechnique internationale ${ }^{4}$, microphone à conducteur mobile.

En électroacoustique le terme dynamic est donc polysémique : tantôt il possède une valeur générique et il doit alors se rendre par électrodynamique ; tantôt il désigne spécifiquement le microphone à bobine mobile. Quant à « microphone dynamique », il est d'un emploi très rare sauf sous la plume de traducteurs trop pressés.

Laissant les microphones à conducteur mobile, on arrive à une troisième catégorie, connue généralement en anglais sous le nom de condenser microphone. Il s'agit de microphones où les variations de pression acoustique se traduisent par une variation de la capacité propre de l'appareil (capacité étant pris ici au sens électrique). L'expression la plus en usage en français pour désigner ce type de microphones semble bien microphone électrostatique, mais on trouve assez fréquemment " microphone à capacité », "microphone à condensateur », tout comme on rencontre en anglais electrostatic et capacitor microphone à côté de condenser. L'important à retenir c'est qu'en Amérique du Nord condenser microphone semble l'appellation la plus courante et qu'elle se rend généralement en français par microphone électrostatique.

On pourrait évidemment étudier les microphones autrement que sous l'angle de leur élément vibrant, comme nous l'avons fait jusqu'ici.

Les propriétés directives sont en effet très souvent prises en considération dans le choix d'un microphone pour une fin précise.

Si le microphone n'est sensible qu'aux ondes sonores ou acoustiques (sound waves) qui proviennent d'une même direction, on le qualifie d'unidirectionnel (unidirectional); s'il possède une sensibilité maximale aux sons provenant de directions diamétralement opposées, on le dit bidirectionnel (bidirectional) et s'il est également sensible aux ondes indépendamment de leur provenance, on le nomme alors omnidirectionnel ou, plus rarement, non directionnel (non-directional or omnidirectional). Les doublets directif et directionnel sont ici en concurrence. Pourtant l'usage tend à spécialiser directif au sens de «qui concerne la directivité » et directionnel au sens de «sensible à la provenance des ondes». C'est ainsi qu'on parlera des effets directifs ${ }^{5}$ ou de propriétés directives, mais on dira des microphones qu'ils sont directionnels.

Pour résumer, schématisons ce bref inventaire terminologique de la façon suivante :

band microphone capacitor microphone condenser microphone

directional effects voir ribbon microphone

voir condenser microphone microphone électrostatique, microphone à condensateur, microphone à capacité effets directifs

4. Décret de 1960, cité par H. Piraux, op. cit., p. 184.

5. H. Piraux, op. cit., p. 187. 
directional microphone dynamic microphone (GB)

dynamic microphone (US) electrostatic microphone (GB)

hum

mike (fam.)

moving-coil microphone non-directional microphone

omnidirectional microphone ribbon microphone

sound wave

unidirectional microphone microphone directionnel

microphone électrodynamique,

microphone à conducteur mobile (normalisé) microphone à bobine mobile

voir condenser microphone ronflement

micro

microphone à bobine mobile

microphone omnidirectionnel, microphone non directionnel

voir non-directional

microphone à ruban

onde sonore (t. courant) onde acoustique ( $t$. technique)

microphone unidirectionnel

Robert DUbuc 